\title{
Quantitative predictions of the $\mu(I)$ rheology in 2D and 3D granular column collapse: scaling laws and quasi-static vs. inertial regimes
}

\author{
Rudy Valette ${ }^{1, *}$, Stéphanie Riber ${ }^{1}$, and Elie Hachem ${ }^{1}$ \\ ${ }^{1}$ MINES ParisTech, PSL Research University, CEMEF, CNRS UMR 7635, CS 10207 rue Claude Daunesse, 06904 Sophia-Antipolis \\ Cedex, France
}

\begin{abstract}
We introduce an accurate numerical method for the computation of 2D and 3D granular collapse under gravity flows using the $\mu(I)$ rheology. We show that this rheology can capture the two experimentally observed types of spreading and the corresponding scaling laws, and we provide an accurate sensitivity analysis to rheological constants. Finally, we underline the role of time-dependency on the spreading dynamics.
\end{abstract}

\section{Introduction}

Granular column collapse under gravity (Fig. 1) is a benchmark problem for several applications from geophysics to industry.

It has been shown experimentally, by Lube et al. [1] for $2 \mathrm{D}$ and Lube et al. [2] and Lajeunesse et al. [3] for 3D granular columns of initial aspect ratio $a=h_{i} / r_{i}$ (initial height/initial width or radius), that for large enough values of $a$, the final width or radius $r_{\infty}$, reached after spreading, obeys the scaling law $\left(r_{\infty^{-}} r_{i}\right) / d_{i} \approx a^{2 / 3}$ in $2 \mathrm{D}$ and $a^{1 / 2}$ in 3D flows. Moreover, it was shown [1] that the normalized distance-time data $(t, x)$ plot (see Fig. 1) exhibits a universal shape, independently of the grain type (fine or coarse sand, rice, sugar, ...).

For shear flows in the dense granular regime, da Cruz et al. [4] shown, by dimensional arguments on discrete numerical models, that the inertial number $I=|\dot{\gamma}| d \sqrt{\rho_{s} / p}$ (where $|\dot{\gamma}|, d, \rho$ and $p$ are the norm of the strain rate tensor, the grain diameter, the grain density and the pressure) governs the transition between a low friction quasi-static regime and a high friction inertial regime.

\section{Flow model and numerical method}

Then, Jop et al. [5] proposed a (computationally lighter) pressure $p$ dependent viscoplastic continuum model called $\mu(I)$, where the extra-stress $\tau_{i j}=(\mu(I) p /|\dot{\gamma}|) \dot{\gamma}_{i j}$ is defined using an effective friction coefficient $\mu(I)=\mu_{s}+\left(\mu_{F}-\mu_{s}\right) /\left(I_{0} / I+1\right)$ that varies from $\mu_{s}$ (quasi-static regime) to $\mu_{F}$ (inertial regime) when the inertial number reaches the (material dependent) parameter $I_{0}$. So far, the $\mu(I)$ model has been successfully applied for the prediction of benchmark steady experimental flows [6]. Concerning complex transient flows of granular column collapse, even though recent papers have shown that discrete numerical simulations can match the experimental scalings $[7,8]$, few studies have addressed the pertinence of the $\mu(I)$ continuum model in 2D [8] and, to our knowledge, 3D studies are still missing.

In this paper, we study the pertinence of the $\mu(I)$ model to predict the case of $2 \mathrm{D}$ and $3 \mathrm{D}$ granular column collapse flows. We use a time-dependent regularization algorithm to solve this model using a finite element method, combined with anisotropic mesh adaptation to capture accurately the quasi-static vs. inertial flow zones, and using a variational multiscale method [9].

A Level-Set method, based on self-reinitialization of the signed distance function, aims to capture and follow efficiently the interface between the fluid/air domains.

\section{Results}

\subsection{Column collapse simulations}

Our simulations show that the $\mu(I)$ model can reproduce all the qualitative features of the experimentally observed flows (see Fig. 1), such as spreading, front flow acceleration, steady and deceleration phases, as well as a remaining flat top for low aspect ratios and the appearance of surface waves for high aspect ratios. Our computations have been validated using a comparison to 2D continuum and discrete simulations from [8], in particular an accurate deceleration phase is predicted (see Fig. 2).

Moreover, 2D and 3D results show that most of the flow is in a quasi-static regime, the inertial regime being only reached in a small volume at the front flow. However, this thin and low pressure front flow contributes

\footnotetext{
* Corresponding author: rudy.valette@mines-paristech.fr
} 
significantly to the spreading distance, in the deceleration phase. 2D and 3D initial aspect ratio scaling laws for spreading distance are then quite accurately retrieved: exponents 0.97 and 0.69 in $2 \mathrm{D}$, and exponents 1.00 and 0.54 in 3D (see Fig. 3 and Fig. 4).

\subsection{Shear bands}

For large friction coefficients $\mu_{s}$, the $\mu(I)$ model exhibits non-unique solutions at early times of the flow (acceleration phase): mesh dependent short length scale instabilities (shear bands) appear, which is consistent with the theoretical predictions of [10]. However, these instabilities do not subsist during the steady front velocity and deceleration phases.

\subsection{Sensitivity to rheological constants}

Sensitivity analysis on rheological constants was performed for quasi-static $\mu_{s}$ and inertial $\mu_{F}$ friction parameters, in order to verify the universality (no dependency on grain type) of flow features such as relative spreading vs. aspect ratio, and normalized distance-time.

As shown on Fig. 5, sensitivity to quasi-static friction is of order $\sim 1 / \mu_{s}$ for low and high aspect ratios. The whole final surface shape is significantly affected, as shown on Fig. 6, where the final global slope is strongly dependent on $\mu_{s}$.

Sensitivity to the inertial friction parameter is subtler (of order $\sim 1 / \mu_{F}$ ), but noticeable (see Fig. 7). As expected, the final surface shape is changed only at larger radii (see Fig. 8), as it was pointed out that the inertial regime is reached at the front flow.

An additional sensitivity, not discussed in experimental papers, is due to the time-dependency. Indeed, the time scale of the flow being $\sim \sqrt{h_{i} / g}$ (where $g$ is the gravity acceleration), a collapse from a larger initial height $h_{i}$, for fixed materials properties and aspect ratio, should benefit to the inertial regime, as the strain rate would scale as $\sim 1 / \sqrt{h_{i}}$ and the pressure as $\sim h_{i}$. Consequently, the inertial number would scale as $\sim 1 / h_{i}$, which would mean a lower friction for larger columns, keeping the aspect ratio constant. A similar effect on inertial number can be deduced by considering the grain diameter (which is an internal length scale not taken into account in the experimental results) rescaled by the column height. Indeed, our computations show that when increasing the initial height, one still get a power law $\sim a^{1 / 2}$ with aspect ratio, but also a larger relative spreading (see Fig. 4, red dots).

\section{Conclusion}

A quantitative dependency of granular collapse flow features to material (rheological) constants was computed in the light of the $\mu(I)$ rheology. This gives complementary information to experimental literature, which reported these features in log plots, where a subtle but real sensitivity exists. Moreover, we have pointed out that the relative spreading should be time-dependent. This property will be investigated in a future experimental study.

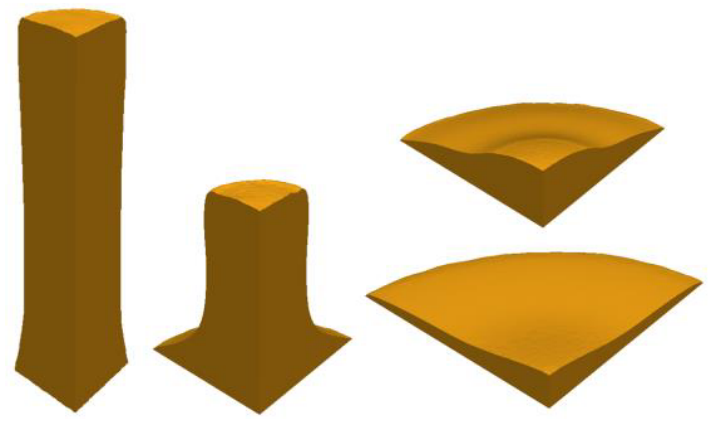

Fig. 1. $\mu(I)$ computation of the shape of a granular column at time $t=20 \%, 40 \%, 60 \%$ and $80 \%$ of total collapse, for an initial ratio $a=7$; rheological parameters were taken from [5]

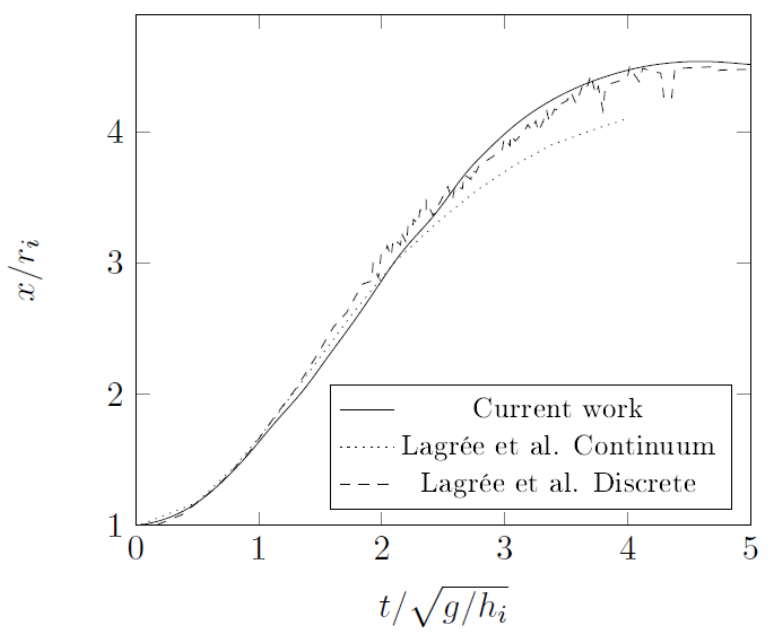

Fig. 2. Normalized distance-time computations: current work $\mu(I)(-)$; Lagrée et al.[8] $\mu(I)$ (..) ; Lagrée et al. [8] 


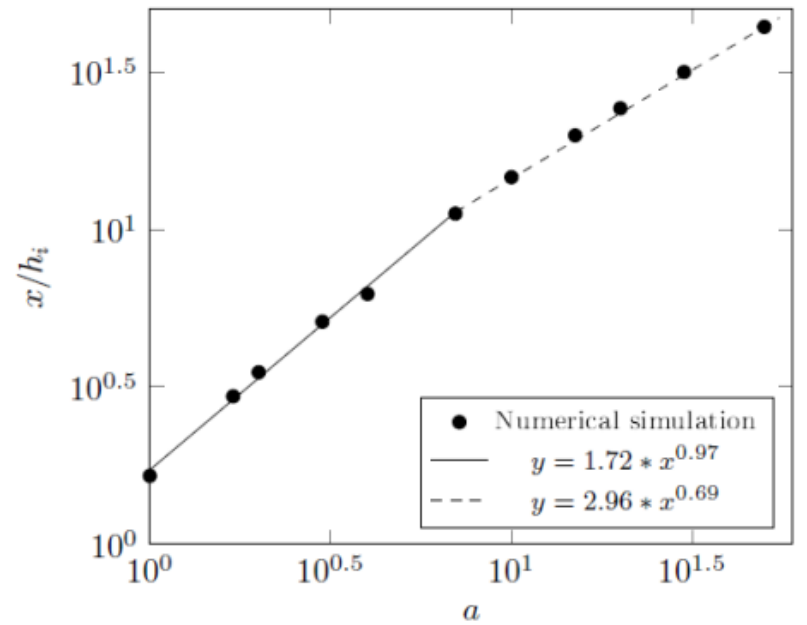

Fig. 3. Relative spreading distance vs. initial aspect ratio for 2D computations

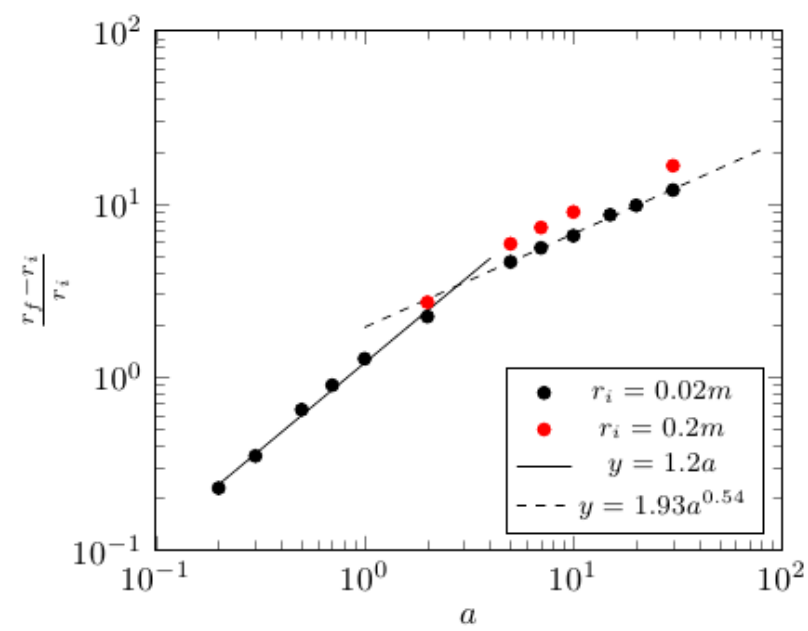

Fig. 4. Relative spreading distance vs. initial aspect ratio for 3D computations - Influence on the initial column height (for each value of aspect ratio $a$, two different radii give two different column heights)

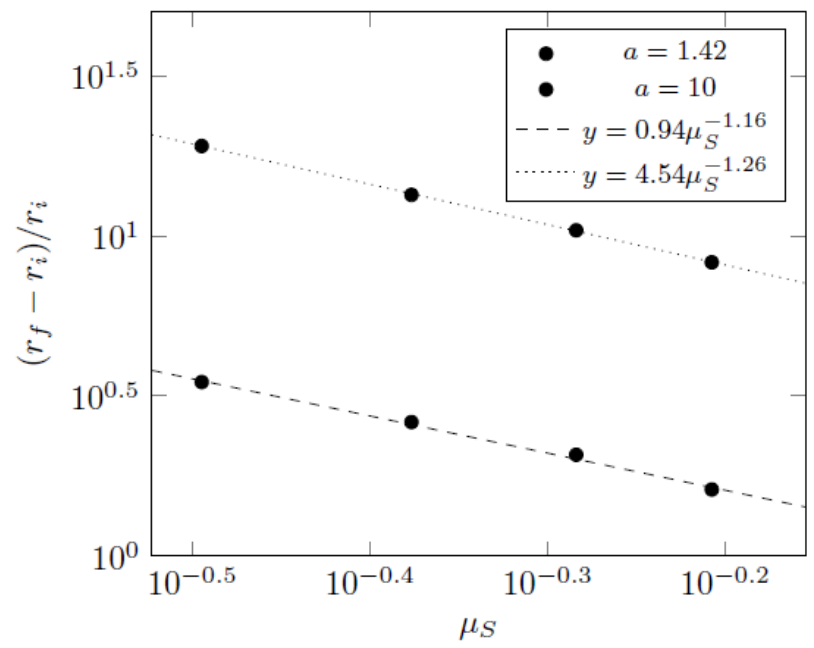

Fig. 5. Variation of relative spreading with quasi-static friction coefficient for different aspect ratios

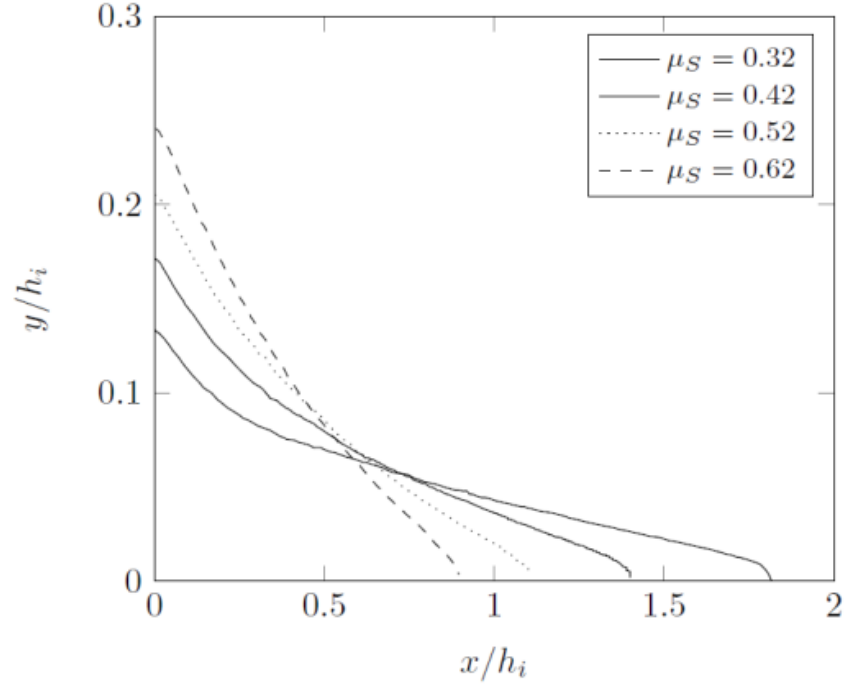

Fig. 6. Variation of final surface with quasi-static friction coefficient for an aspect ratio $a=10$

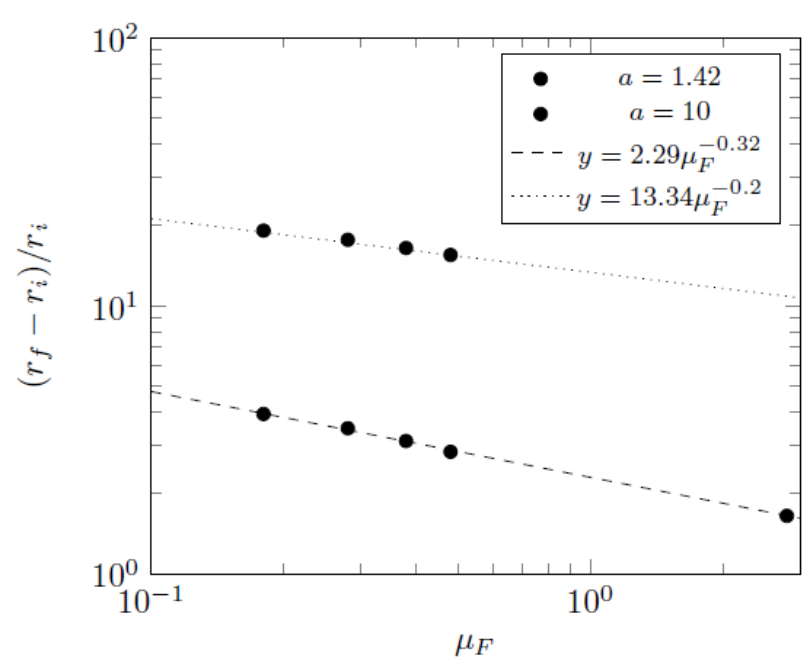

Fig. 7. Variation of relative spreading with inertial friction coefficient for different aspect ratios

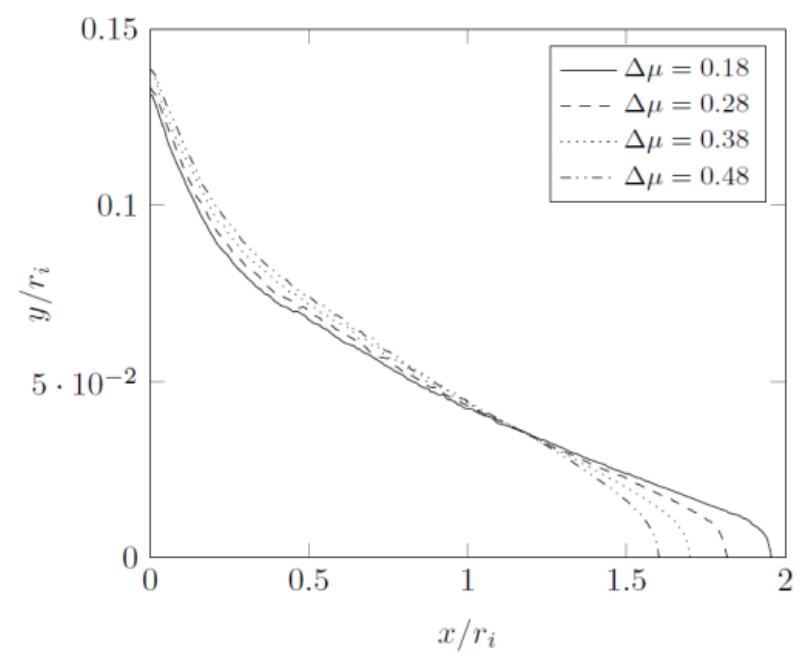

Fig. 8. Variation of final surface with inertial friction coefficient for an aspect ratio $a=10$ 


\section{References}

1. G. Lube, H. E. Huppert, R. S. J. Sparks, A. Freundt, Phys. Rev.E, 72, 041301 (2005)

2. E. Lajeunesse, A.Mangeney-Castelnau, J. P. Vilotte, Phys. Fluids, 16, 2371 (2004)

3. G. Lube, H. E. Huppert, R. S. J. Sparks, M. A. Hallworth, J. Fluid. Mech., 508, 175 (2004)

4. F. da Cruz, S. Emam, M. Prochnow, J.-N. Roux, F. Chevoir ,Phys. Rev. E, 72, 021309 (2005)

5. P. Jop, O. Pouliquen, Y. Forterre, Nature, 441, 727 (2006)

6. G.D.R. Midi, Eur. Phys. J. E, 14, 341 (2004)

7. L. Lacaze, R. R. Kerswell, Phys. Rev. Let., 102, 108305 (2009)

8. P.-Y. Lagrée, L. Staron, S. Popinet, J. Fluid Mech., 686, 378 (2011)

9. S. Riber, R. Valette, Y. Mesri, E. Hachem, Computers and Fluids, 138, 51 (2016)

10. T. Barker, D.G. Schaeffer, P. Bohorquez, J. M. N.

T. Gray, J. Fluid Mech., 779, 794 (2015) 